\title{
Promoting Bone Health in MPS VI (Maroteaux-Lamy syndrome): Framing New Therapies, Part 2
}

Welcome to the second issue on mucopolysacharide (MPS) disease with focus on bone health. This issue, as was the case for the preceding issue, is based on an international meeting of experts held at Children's Hospital and Research Center Oakland entitled "Promoting Bone Health in MPS VI: Framing New Therapies". The meeting was jointly sponsored by Children's Hospital and Research Center Oakland and the Department of Pediatrics, University of Padova, Italy. Editorial assistance was provided by Helen Nicely, PhD, BioMarin, and Ismar Health Care NV, Belgium and was paid for by BioMarin.

The MPS diseases are a group of relatively rare genetic disorders that involve deficiency of a specific lysosomal enzyme required to complete glycosaminoglycan degradation in the lysosome. They consist of MPS I (Hurler syndrome), MPS II (Hunter syndrome), MPS IIIA-D (Sanfilippo syndrome), MPS IV(A\&B) (Morquio syndrome), and MP VI (Maroteaux-Lamy syndrome), MPS VII (Sly syndrome), and MPS IX. Table 1 summarizes the disorders by name, eponym, enzyme deficiency and specific glycosaminoglycans stored in each syndrome. For many of us, when we first started our careers and/or training, the treatments for this group of diseases were considered primarily supportive or palliative.

This issue contains original research articles, clinical reviews, commentary, and basic science reviews examining mechanisms for the bone disease, diagnosis, monitoring and treatment in MPS patients. In their article, Decker et al., report on the effect of enzyme replacement therapy (ERT) with galsulfase (recombinant human $N$-acetylgalactosamine 4-sulfatase) on growth and puberty and demonstrate positive effects on both important parameters in MPS VI patients. White et al., in the second original research paper, use the Pediatric Outcomes Data Collection Instrument (PODCI) to demonstrate improvement in musculoskeletal health in MPS II patients after 1 year of enzyme replacement therapy (recombinant human I2S). PODCI is a validated, functional measure of musculoskeletal health in children with disabilities. Alliston in her article "Chondroitin sulfate and growth factor signaling in the skeleton: Possible links to MPS VI(Maroteaux-Lamy syndrome)", explores the hypothesis that accrued partially degraded glycosaminoglycans (GAGs) may contribute to deregulation of signaling pathways that normally orchestrate skeletal development. McDonald et al. in "Clinical utility of endurance measures for evaluation of treatment in patients with mucopolysaccharidosis VI (Maroteaux-Lamy syndrome)" describe the use of the 6-MWT (minute walk test), 12-MWT and 3-MSC (minute stair climb) as measures of endurance to evaluate MPS patient response to specific enzyme replacement therapy. In "Radiologic and Neuroradiologic Findings in the Mucopolysaccharidoses", Lachman et al. discuss the skeletal X-ray and neuroimaging findings in MPS patients and the imaging techniques that can be used for diagnosing and monitoring abnormalities in the skeleton and central nervous system. Finally, Wilson and Brömme in their article "Potential Role of Cathepsin K in the Pathophysiology of Mucopolysaccharidoses" propose a second basic science mechanism involving the inhibition of osteoclast function through altered activity of cathepsin K by GAGs for the bone disease seen in these patients.

As this and the previous issue demonstrate, the identification and characterization of the responsible en- 
Table 1

Biochemical classification of the mucopolysaccharide disorders

\begin{tabular}{llll}
\hline Number* & Eponym & Enzyme deficiency & Glycosaminoglycan stored \\
\hline MPS I (severe) & Hurler syndrome & $\alpha$-L-iduronidase & Dermatan sulfate, heparan sulfate \\
MPS I (attenuated) & Scheie syndrome & $\alpha$-L-iduronidase & Dermatan sulfate, heparan sulfate \\
MPS I (attenuated) & Hurler-Scheie syndrome & $\alpha$-L-iduronidase & Dermatan sulfate, heparan sulfate \\
MPS II (severe) & Hunter (severe) syndrome & Iduronate sulfatase & Dermatan sulfate, heparan sulfate \\
MPS II (attenuated) & Hunter (mild) syndrome & Iduronate sulfatase & Dermatan sulfate, heparan sulfate \\
MPS IIIA & Sanfilippo A syndrome & Heparan N-sulfatase & Heparan sulfate \\
MPS IIIB & Sanfilippo B syndrome & $\alpha$-N-acetyl-glucosaminidase & Heparan sulfate \\
MPS IIIC & Sanfilippo C syndrome & Acetyl CoA: $\alpha$-gulcosaminide & Heparan sulfate \\
& & acetlytransferase & Heparan sulfate \\
MPS IIID & Sanfilippo D syndrome & N-acetylglucosamine 6-sulfatase & Keratan sulfate, chondroitin 6-sulfate \\
MPS IVA & Morquio syndrome, type A & Galactose-6-sulfatase & Keratan sulfate \\
MPS IVB & Morquio syndrome, type B & $\beta$-galactosidase & Dermatan sulfate \\
MPS VI & Maroteaux-Lamy syndrome & N-acetylgalactosamine 4-sulfatase & (arylsulfatase B) \\
MPS VII & & $\beta$-glucuronidase & Dermatan sulfate, heparan sulfate \\
& Sly syndrome & & Hyondroitin 4-, 6-sulfates \\
MPS IX & & Hyaluronidase & Hyaluronan \\
\hline
\end{tabular}

*Note that MPS type designations V and VII are no longer used.

From: Joseph Muenzer, The mucopolysaccharidoses: A heterogeneous group of disorders with variable pediatric presentations, Pediatrics 144 (2004), S27-S34

zyme for each disease, cloning of the respective genes, and development of in vitro systems for large scale production and isolation of these enzymes has led to development of enzyme replacement therapy for MPS I, II and VI. Availability of these specific therapies has led us to "expand" our therapeutic horizons and expectations with the specific goals of providing enhanced functional capabilities, improved quality of life and potentially a longer life expectancy for MPS patients. These issues emphasize the importance of translational research and the transfer of basic science discoveries to therapeutic interventions for rare clinical problems such as MPS. With the advent of enzyme replacement therapy, the hope is to expand the treatment options for many more patients.

\author{
Jacob Neufeld, MD \\ Editor-in-Chief
}

\section{Conflict of interest}

Dr. Neufeld received an honorarium for his lecture at the conference. 